\title{
Mobility Effects on the Performance of Mobile Ad hoc Networks
}

\begin{abstract}
In this paper, we present the implementation and analysis of our implemented testbed considering the Optimized Link State Routing (OLSR) protocol for Mobile Ad hoc Networks (MANET). We investigate the effect of mobility and topology changing in the throughput of MANETs. We study the impact of best-effort traffic for Mesh Topology. Experimental time is 150 seconds. In this work, we consider 6 experimental models and we assess the performance of our testbed in terms of throughput, round trip time and packet loss. From our experiments, we found that the OLSR protocol has a good performance when the source node is moving. However, the performance is not good when the three relay nodes are moving.
\end{abstract}

\section{Introduction}

A Mobile Ad hoc Network (MANET) is a collection of wireless mobile terminals that are able to dynamically form a temporary network without any aid from fixed infrastructure or centralized administration. The growth of laptops and wireless networking have made MANET a popular subject for research since the mid to late 1990s. In recent years, MANET are continuing to attract the attention for their potential use in several fields. Mobility and the absence of any fixed infrastructure make MANET very attractive for mobility and rescue operations and time-critical applications. A typical example of this approach has revealed many aspects of IEEE 802.11, like the gray-zones effect [1], which usually are not taken into account in standard simulators, as the well-known $n s$ - 2 simulator. So far we can count a lot of simulation results on the performance of MANET, e.g. in terms of end-to-end throughput, delay and packet loss. However, in order to assess the simulation results, real-world experiments are needed and a lot of testbeds have been built to date $[2,3,4,5]$. The baseline criteria usually used in realworld experiments is guaranteeing the repeatability of tests, i.e. if the system does not change along the experiments. How to define a change in the system is not a trivial problem in MANET, especially if the nodes are mobile.

In this paper, we concentrate on the performance analysis of a small testbed of five laptops acting as nodes of a MANET. We use Optimized Link State Routing (OLSR) protocol, which is a pro-active routing protocol, and it has been gaining great attention within the scientific commu- 
nity. Furthermore, the olsrd [6] software we have used in our experiments is the most updated software we have encountered.

In our previous work, we found the following results. We proved that while some of the OLSR's problems can be solved, for instance the routing loop, this protocol still have the self-interference problem. There is an intricate interdependence between MAC layer and routing layer, which can lead the experimenter to misunderstand the results of the experiments. For example, the horizon is not caused only by IEEE 802.11 Distributed Coordination Function (DCF), but also by the routing protocol. We carried out the experiments considering stationary nodes of ad-hoc network. We also considered the node mobility and carry out experiments for OLSR and B.A.T.M.A.N. protocols [7]. We found that throughput of TCP were improved by reducing Link Quality Window Size (LQWS), but there were packet loss because of experimental environment and traffic interference. For TCP data flow, we got better results when the LQWS value was 10. Moreover, we found that the node join and leave operations affect more the TCP throughput and RTT than UDP [8].

In this work, we investigate the topology changing of MANET. We implemented six MANET models and carried out experiments for different topologies.

The structure of the paper is as follows. In Section 2, we present the related work. In Section 3, we present the testbed design and implementation. In Section 4, we present experimental evaluation. Finally, conclusions are given in Section 5.

\section{Related Work}

In [9], the authors analyze the performance of an outdoor ad-hoc network, but their study is limited to reactive protocols such as Ad hoc On Demand Distance Vector (AODV) [10] and Dynamic Source Routing (DSR) [11]. The authors of [12], performs outdoor experiments of non standard proactive protocols. Other ad-hoc experiments are limited to identify MAC problems, by providing insights on the onehop MAC dynamics as shown in [13].

The closest work to ours is that in [14]. However, the authors did not care about the routing protocol. In [15], the disadvantage of using hysteresis routing metric is presented through simulation and indoor measurements. Our experiments are concerned with the interaction of transport protocols and routing protocol, for instance OLSR. Furthermore, we compare the performance of the testbed for six scenarios.

In [16], the authors presents an experimental comparison of OLSR using the standard hysteresis routing metric and the Expected Transmission Count (ETX) metric in a 7 by 7 grid of closely spaced Wi-Fi nodes to obtain more realistic results. The throughput results are similar to our previous work and are effected by hop distance [17].

\section{Testbed Design and Implementation}

\subsection{Target Environment}

We have implemented a MANET testbed which provides a realistic platform for analyzing various aspect of these networks, including the different topology models. For our testbed, we make the following considerations.

- We consider an indoor environment at our departmental floor.

- We investigate the effect of mobility and topology changing in the throughput of MANET testbed.

- We constructed six experimental models: Model 1 (only one relay node is moving); Model 2 (only one source node is moving); Model 3 (two relay nodes are moving); Model 4 (one relay node and one source node are moving); Model 5 (all relay nodes are moving); Model 6 (one source node and two relay node are moving).

- The mobile nodes move toward the destination at a regular speed. When the mobile nodes arrive at the corner, they stop for about three seconds.

- In order to make the experiments easier, we implemented a testbed interface and web tool.

- Experimental time is 150 seconds.

\subsection{OLSR Overview}

The link state routing protocol that is most popular today in the open source world is OLSR from olsr.org. OLSR with Link Quality (LQ) extension and fisheye-algorithm works quite well. The OLSR protocol is a pro-active routing protocol, which builds up a route for data transmission by maintaining a routing table inside every node of the network. The routing table is computed upon the knowledge of topology information, which is exchanged by means of Topology Control (TC) packets. OLSR makes use of HELLO messages to find its one hop neighbors and its two hop neighbors through their responses. The sender can then select its Multi Point Relays (MPR) based on the one hop node which offer the best routes to the two hop nodes. By this way, the amount of control traffic can be reduced. Each node has also an MPR selector set which enumerates nodes that have selected it as an MPR node. OLSR uses TC messages along with MPR forwarding to disseminate neighbor 


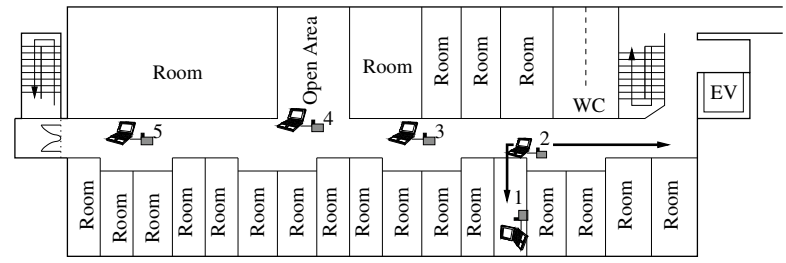

Figure 1. MV1 model.

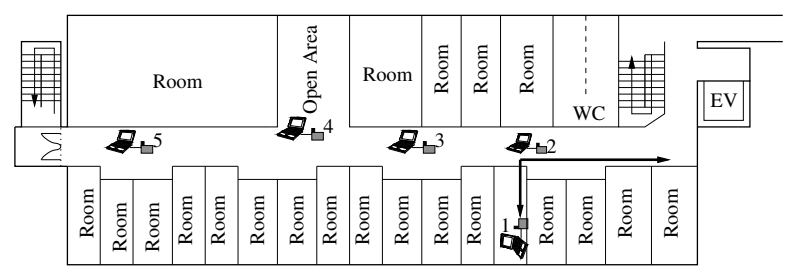

Figure 2. MV2 model.

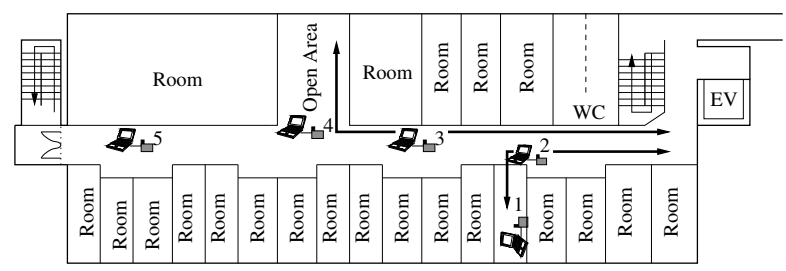

Figure 3. MV3 model.

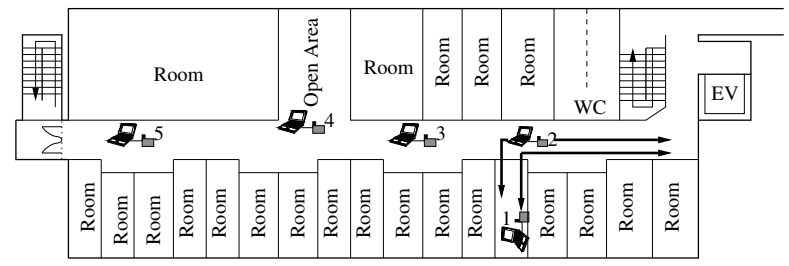

Figure 4. MV4 model.

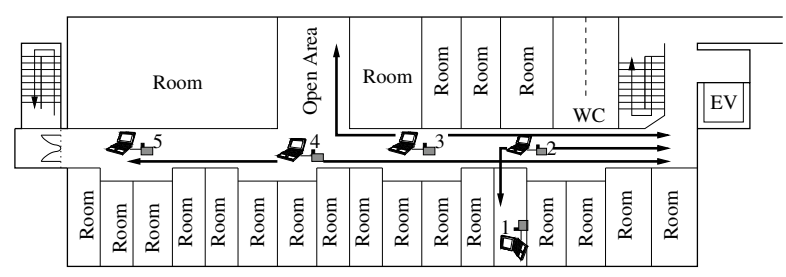

Figure 5. MV5 model.

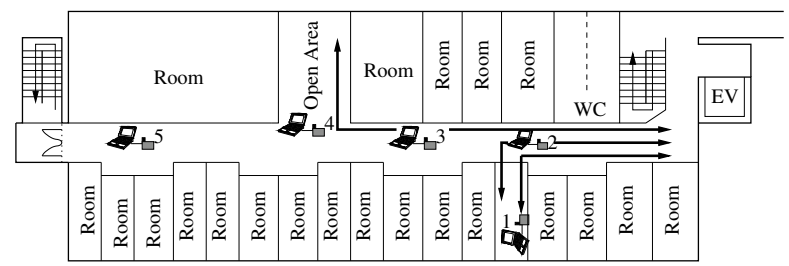

Figure 6. MV6 model.
Table 1. Classification of nodes for each experimental model.

\begin{tabular}{c|c|c|c}
\hline Model & \multicolumn{2}{|c|}{ Number of moving nodes } & Num. of stati- \\
& Source node & Relay node & onary nodes \\
\hline \hline MV1 & 0 & 1 & 4 \\
MV2 & 1 & 0 & 4 \\
MV3 & 0 & 2 & 3 \\
MV4 & 1 & 1 & 3 \\
MV5 & 0 & 3 & 2 \\
MV6 & 1 & 2 & 2 \\
\hline
\end{tabular}

information throughout the network. Host Network Address (HNA) messages are used by OLSR to disseminate network route advertisements in the same way TC messages advertise host routes.

OLSRv2 is currently being developed at IETF. It maintains many of the key features of the original protocol including MPR selection and dissemination. Key differences are the flexibility and modular design using shared components such as packet format packetbb and neighborhood discovery protocol.

In our OLSR code, a simple RFC-compliant heuristic is used [18] to compute the MPR nodes. Every node computes the path towards a destination by means of a simple shortest-path algorithm, with hop-count as target metric. In this way, a shortest path can result to be also not good, from the point of view of the packet error rate. Accordingly, recently olsrd has been equipped with the LQ extension, which is a shortest-path algorithm with the average of the packet error rate as metric. This metric is commonly called as the ETX, which is defined as $\operatorname{ETX}(i)=$ $1 /(N I(i) \times L Q I(i))$. Given a sampling window $W, \mathrm{NI}(i)$ is the packet arrival rate seen by a node on the $i$-th link during $W$. Similarly, LQI $(i)$ is the estimation of the packet arrival rate seen by the neighbor node which uses the $i$-th link. When the link has a low packet error rate, the ETX metric is higher. The LQ extension greatly enhances the packet delivery ratio with respect to the hysteresis-based technique [19].

\subsection{Testbed Description}

Our testbed is composed of five laptops. We constructed six experimental models as shown in Figs. $1-6$. The experimental parameters are shown in Table 1. In Fig. 1, only one relay node (node 2 ) is moving. In the second model only one source node (node 1) is moving (see Fig. 2). The mobile node moves toward the destination at a regular speed. When the mobile node arrives at the corner, it stops for about three seconds. The round-trip time is 50 seconds. We 
Table 2. Experimental parameters.

\begin{tabular}{c|c}
\hline Function & Value \\
\hline \hline Number of Nodes & 5 \\
MAC & IEEE 802.11 \\
Packet Rate & $122 \mathrm{pps}$ \\
Number of Trials & 5 \\
Packet Size & 512 bytes \\
Duration & $150000 \mathrm{~ms}$ \\
Protocol & OLSR \\
LQ Window Size & 10 \\
\hline
\end{tabular}

call this model MV2. In the third model, two relay nodes are moving (node 2 and node 3 ) as shown in Fig. 3. We call this model MV3. In the forth model, source node and one relay node (node 2 ) are moving as shown in Fig. 4. We call this model MV4. In Fig. 5, all relay nodes (node 2, node 3 and node 4) are moving. We call this model MV5. In Fig. 6 , source node and two relay node are moving. We call this model MV6 ${ }^{1}$. The round-trip time of node 3 is about 80 seconds.

The operating system mounted on these machines is Fedora Core 4 Linux with kernel 2.6.x, suitably modified in order to support the wireless cards. The wireless network cards are from Linksys. They are usb-based cards with and external antenna of $2 \mathrm{dBi}$ gain, transmitted power of $16+/-$ $1 \mathrm{dBm}$ and receive sensitivity of $-80 \mathrm{dBm}$. We verified that the external antenna improves the quality of the first hop link, which is the link connecting the ad-hoc network.The driver can be downloaded from the web site in reference [20].

The source node used to coordinates the measurement campaign, as well as graphical tools to check network connectivity. In our testbed, we have two systematic background or interference traffic we could not eliminate: the control traffic and the other wireless Access Points (APs) interspersed within the campus. The control traffic is due to the ssh program, which is used to remotely start and control the measurement software on the source node. The other traffic is a kind of interference, which is typical in an academic scenario.

\subsection{Testbed Interface}

Until now, all the parameters settings and editing were done by using command lines of bash shell (terminal), which resulted in many misprints and the experiments were repeated many times. In order to make the experiments easier, we implemented a testbed interface. For the Graphical User Interface (GUI) we used wxWidgets tool and each operation is implemented by Perl language. wxWidgets is a

\footnotetext{
${ }^{1}$ In MV2, MV4 and MV6, the source node is always moving.
}

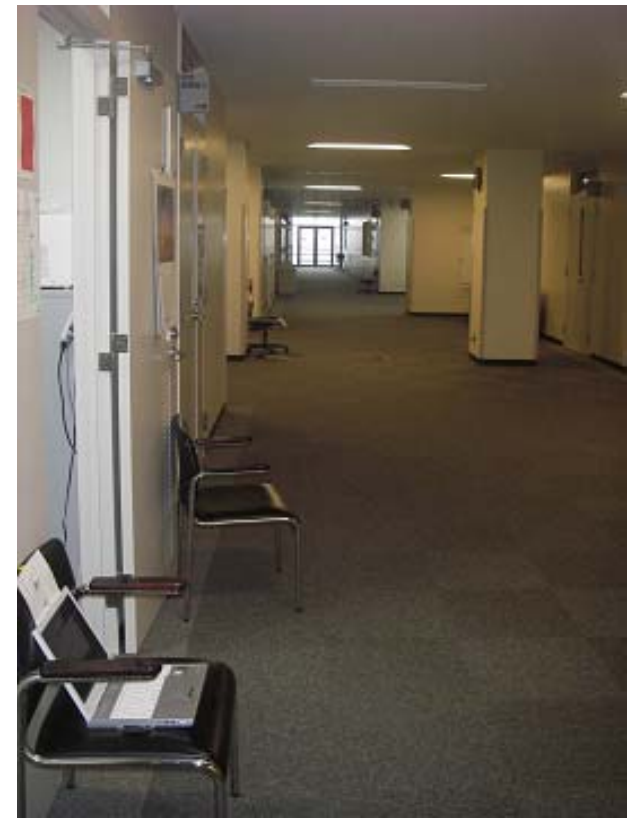

Figure 7. Snapshot of indoor environment.

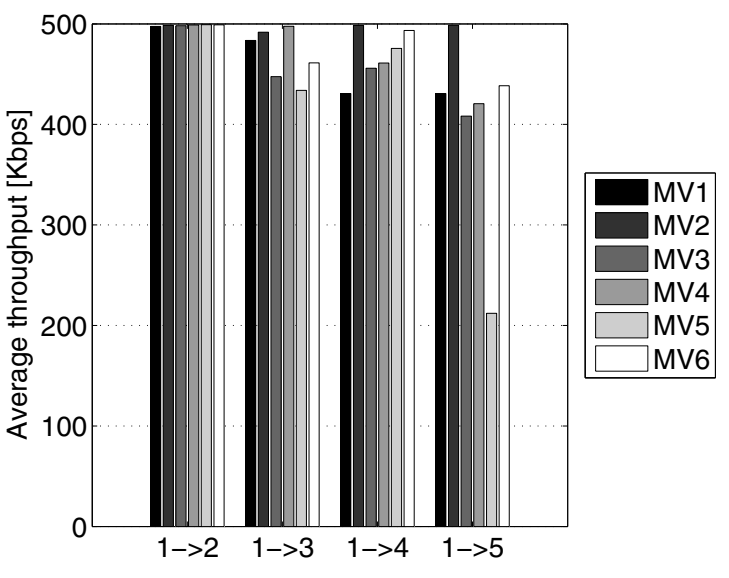

Figure 8. Comparison of average throughput using UDP data flow.

cross-platform GUI and tools library for GTK, MS Windows and Mac OS X.

We implemented many parameters in the interface such as transmission duration, number of trials, source address, destination address, packet rate, packet size, LQWS, and topology setting function. We can save the data for these parameters in a text file and can manage in a better way the experimental conditions. Moreover, we implemented collection function of experimental data in order to make easier the experimenter's work. 


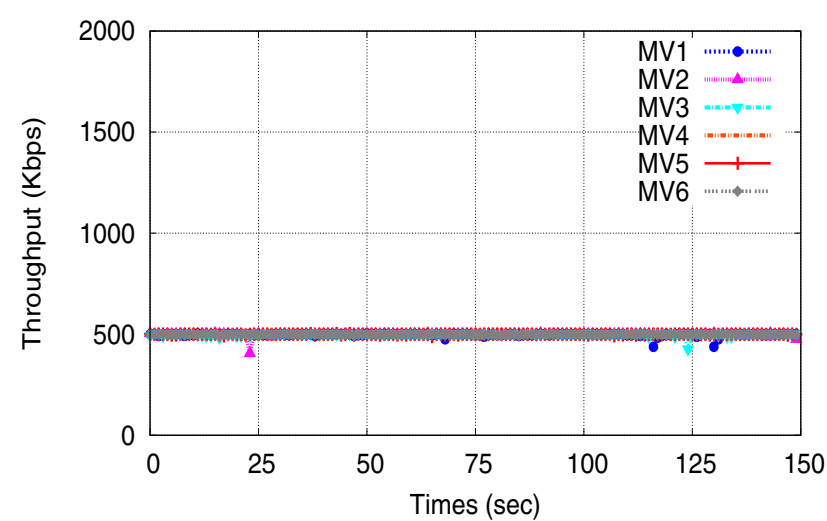

(a) UDP, $1 \rightarrow 2$

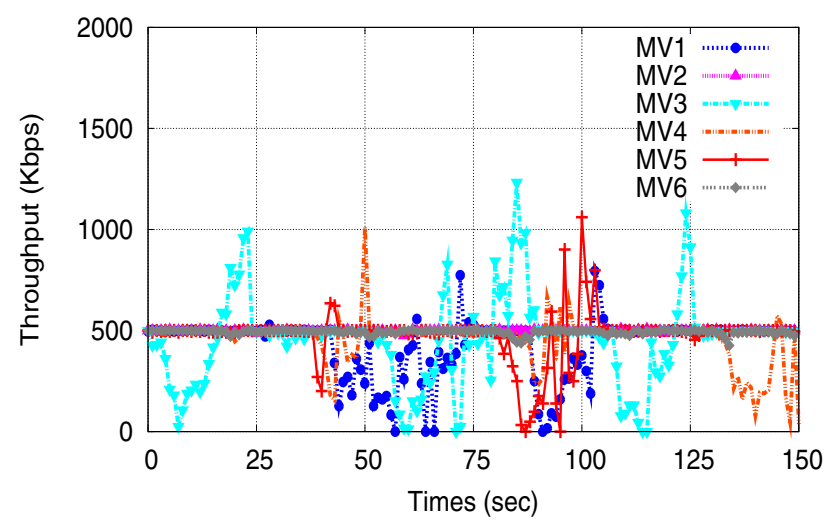

(c) UDP, $1 \rightarrow 4$

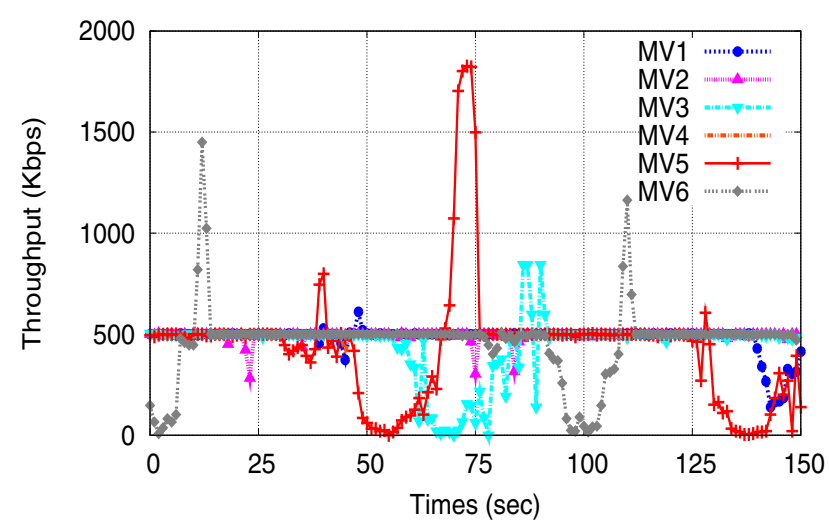

(b) UDP, $1 \rightarrow 3$

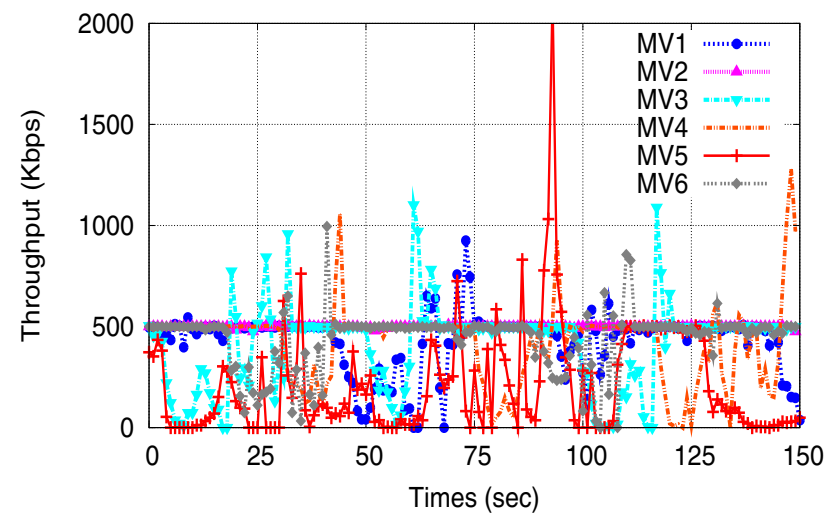

(d) UDP, $1 \rightarrow 5$

Figure 9. Throughput results.

\section{Experimental Results}

\subsection{Experimental Settings}

The experimental parameters are shown in Table 2. A snapshot of the indoor environment for experiments is shown in Fig. 7. We study the impact of best-effort traffic for Mesh Topology (MT). In the MT scheme, the MAC filtering routines are not enabled. We collected data for three metrics: the throughput, Round-Trip Time (RTT) and packet loss. These data are collected by using the Distributed Internet Traffic Generator (D-ITG) [21], which is an open-source Internet traffic generator. D-ITG computes the packet loss as the number of lost packet divided by the effective number of sent packets.

In previous experiments [7, 17, 22], we realized that an external antenna improves radio signal reception. The transmission rate of the data flows is $122 \mathrm{pps}=499.712 \mathrm{Kbps}$, i.e. the packet size of the payload is 512 bytes. All exper- iments have been performed in indoor environment, within our departmental floor of size roughly $100 \mathrm{~m}$. All laptops are in radio range of each other. In our previous work, one experiment lasted about 10 seconds and was repeated 50 times. But, the experimental time was very short. For this reason, in this paper we set the experimental time about 150 seconds. We measured the throughput for UDP and TCP, which is computed at the receiver. We estimated the packet loss to compute the link quality metrics, e.g. LQ. For OLSR, $w T_{\mathrm{HELLO}}<T_{\mathrm{Exp}}$, where $T_{\mathrm{Exp}}$ is the total duration of the experiment, i.e., in our case, $T_{\operatorname{Exp}}=500 \mathrm{~s}$, and $T_{\text {HELLO }}$ is the rate of the HELLO messages. However, the testbed was turned on even in the absence of measurement traffic. Therefore, the effective $T_{\operatorname{Exp}}$ was much greater.

As MAC protocol, we used IEEE $802.11 \mathrm{~b}$. The transmission power was set in order to guarantee a coverage radius equal to the maximum allowed geographical distance in the network. Since we were interested mainly in the performance of the routing protocol, we kept unchanged 


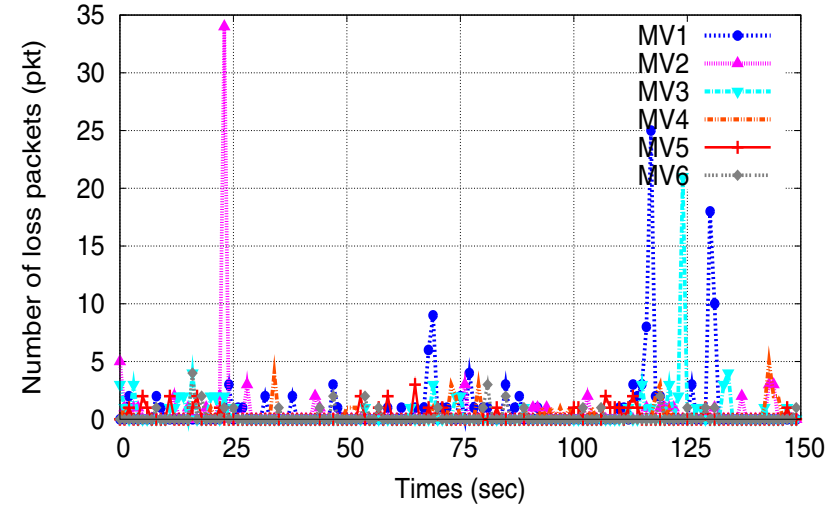

(a) UDP, $1 \rightarrow 2$

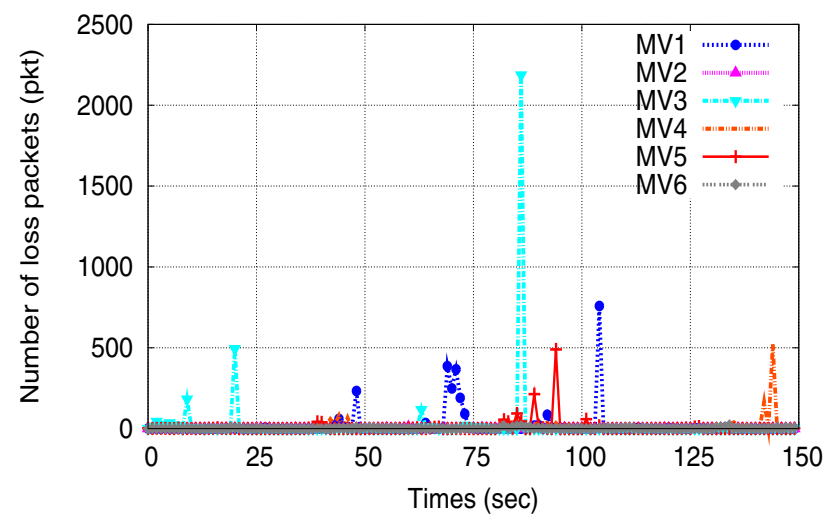

(c) UDP, $1 \rightarrow 4$

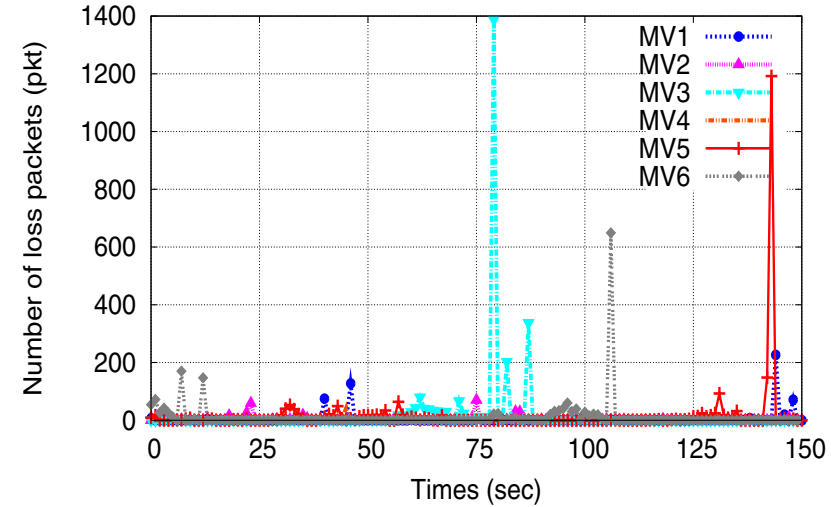

(b) UDP, $1 \rightarrow 3$

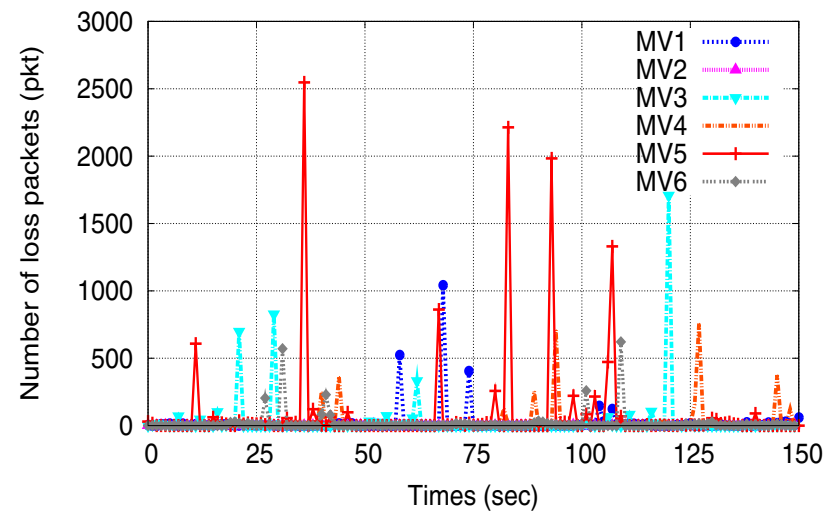

(d) UDP, $1 \rightarrow 5$

Figure 10. Packet loss results.

all MAC parameters, such as the carrier sense, the retransmission counter, the contention window and the RTS/CTS threshold. Moreover, the channel central frequency was set to $2.412 \mathrm{GHz}$ (channel 1). In regard to the interference, it is worth noting that, during our tests, almost all the IEEE 802.11 spectrum had been used by other APs disseminated within the campus. In general, the interference from other access points is a non-controllable parameter.

\subsection{Experimental Measurements}

In order to show the range of variability of the data, we also report the plot of the metrics according to the model types, as shown in Fig. 8. The horizontal axis show the hop distance, i.e. 1->2 means source node id $->$ destination node $i d$. The vertical axis shows the average throughput (Kbps), which is computed at the receiver. As you can see in Fig. 8, when source node is moving, we found that the average throughput was decreased. Especially, it seems that for MV5 model the throughput decreases much more. This is because of the effect of the relay node movement and it was caused by routing loops.

We show the throughput results in Fig. 9. The horizontal axis shows the experimental times (sec). The vertical axis shows the throughput (Kbps), which is computed at the receiver. As shown in Fig. 9 (a), we can see a stable constant bit rate flow for each experimental models. As shown in Fig. 9 (b), there are some oscillations in MV3, MV5 and MV6 models. This is because of movement of node 3.

Moreover, the number of packet loss increases after node $1->4$ in Fig. 10. We can confirm these results also looking at Fig. 11 for RTT. It seems that the topology can be very dynamic in MV3 and MV5. We got a lot of oscillation in MV5 model (as shown in Figs. 11 (d) and 10 (d)). A direct symmetric link exists between nodes 1 and 4, and OLSR chooses correctly the 2-hops route, i.e. 1-2-4 or 1-3-4. In this case, the hop-count threshold is 3 , and, again, we find high variability for the connection $1-4$, which incurs in the longest route, e.g. 1-2-3-4.

From these results, the UDP data flow is affected more in MV5 compared with MV6. We found that OLSR protocol 


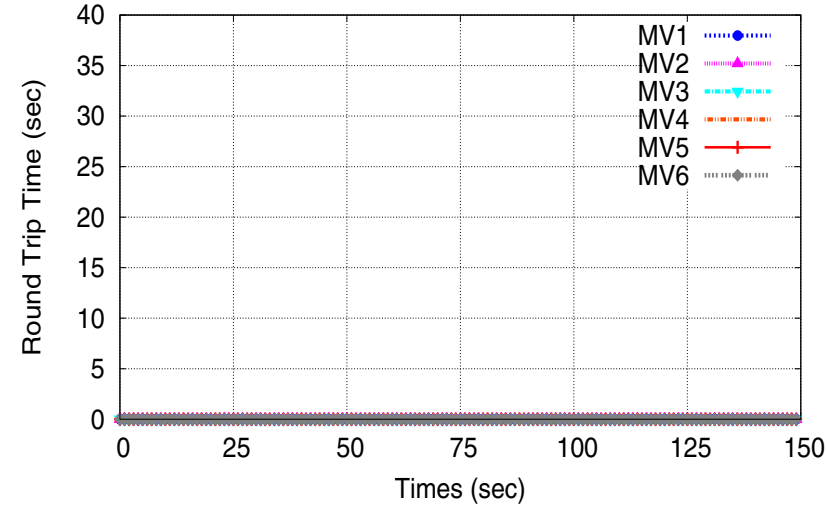

(a) UDP, $1 \rightarrow 2$

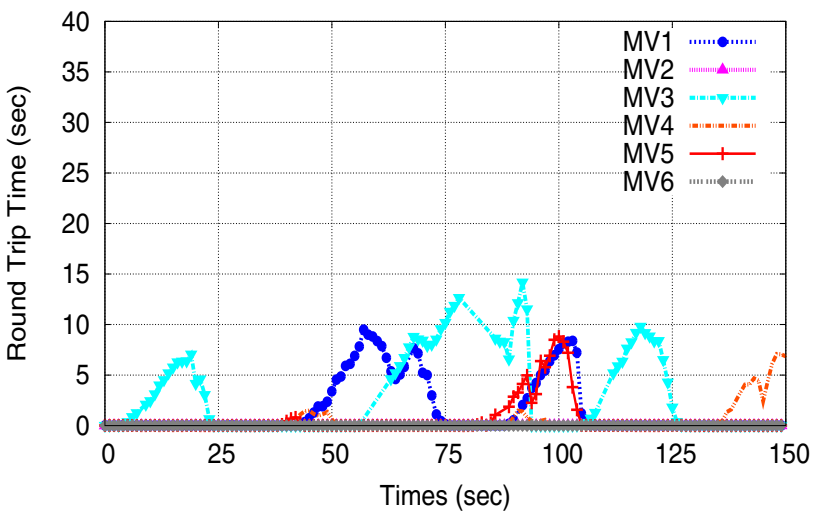

(c) UDP, $1 \rightarrow 4$

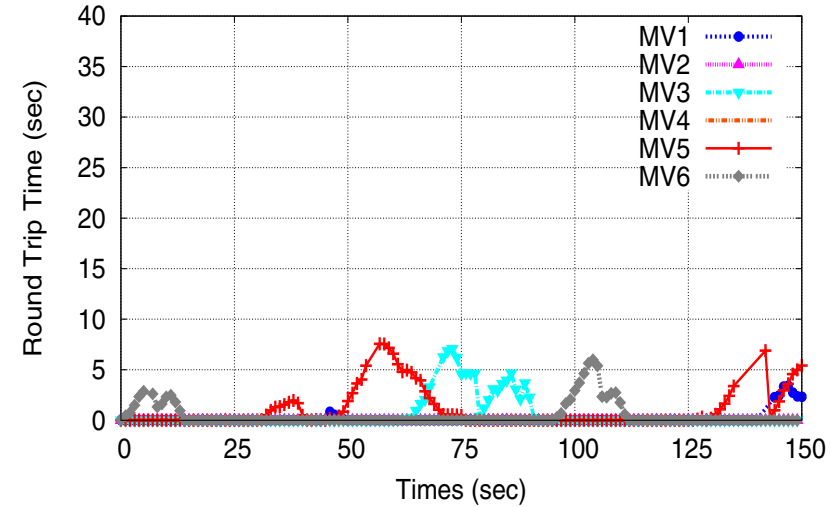

(b) UDP, $1 \rightarrow 3$

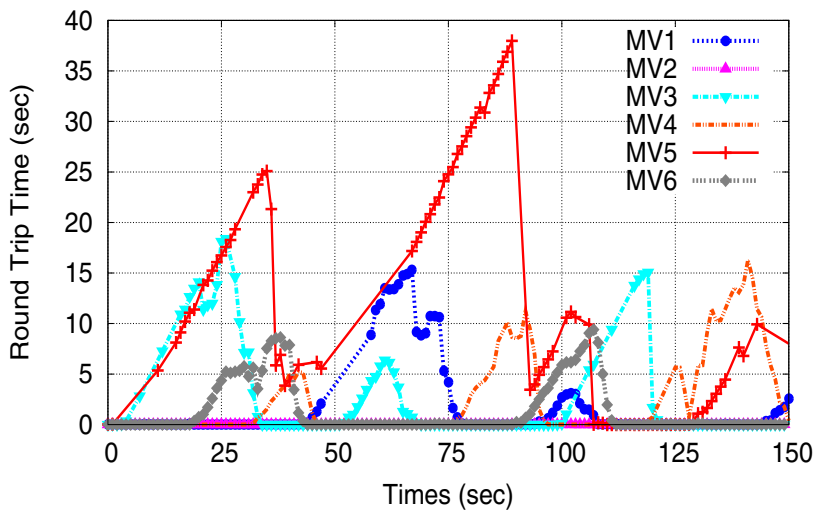

(d) UDP, $1 \rightarrow 5$

Figure 11. RTT results.

has a good performance when the source node is moving. However, the performance is not good when the relay nodes are moving.

\section{Conclusions}

In this paper, we carried out experiments for a small MANET testbed with five nodes. We used OLSR protocol for real experimental evaluation.

In our experiments, we considered six models: MV1, MV2, MV3, MV4, MV5 and MV6. In MV1, only one node is moving (relay node). In MV2, source node is moving. In MV3, two relay nodes are moving. In MV4, one relay node and one source node are moving. In MV5, three relay nodes are moving. In MV6, one source node and two relay nodes are moving. We assessed the performance of our testbed in terms of throughput, RTT and packet loss.

From our experiments, we found the following results.
- There are some oscillations in MV3, MV5 and MV6 models. This is because of movement of node 3 .

- The number of packet loss increases after third hop.

- We found that the OLSR protocol has a good performance when the source node is moving. However, the performance is not good when the relay nodes are moving.

- There are a lot of oscillations in MV5 model.

- The OLSR protocol needs to be equipped with more realistic topology control mechanism in order to be used in different scenarios.

These experiments where performed using a single flow through the network. In the future, we would like to consider the effect of multiple flows on the routing and increase the number of nodes. Moreover, we would like to consider the effect of destination node movement and extension of our testbed. 


\section{Acknowledgment}

This work is supported by a Grant-in-Aid for scientific research of Japan Society for the Promotion of Science (JSPS). The authors would like to thank JSPS for the financial support.

\section{References}

[1] H. Lundgren, E. Nordströ, and C. Tschudin, “Coping with communication gray zones in IEEE $802.11 \mathrm{~b}$ based ad hoc networks," in WOWMOM '02: Proceedings of the 5th ACM international workshop on Wireless mobile multimedia, 2002, pp. 49-55.

[2] W. Kiess and M. Mauve, "A survey on real-world implementations of mobile ad-hoc networks," Ad Hoc Networks, vol. 5, no. 3, pp. 324-339, 2007.

[3] E. Nordström, "Ape - a large scale ad hoc network testbed for reproducible performance tests," Master's thesis, Uppsala University, 2002.

[4] “The freifunk project," http://wiki.freifunk.net/.

[5] R. Draves, J. Padhye, and B. Zill, "Comparison of routing metrics for static multi-hop wireless networks,' in SIGCOMM '04, 2004, pp. 133-144.

[6] A. Tønnesen, "OLSRd: Implementation code of the OLSR,” http://www.olsr.org/.

[7] L. Barolli, M. Ikeda, G. De Marco, A. Durresi, and $\mathrm{X}$. Fatos, "Performance analysis of OLSR and BATMAN protocols considering link quality parameter,' in Proc. AINA 2009, May 2009, pp. 307-314.

[8] M. Ikeda, L. Barolli, M. Hiyama, T. Yang, G. De Marco, and A. Durresi, "Performance evaluation of a manet testbed for differenet topologies," in Proc of NBiS-2009, Indianapolis, Auguest 2009, pp. 327-334.

[9] D. A. Maltz, J. Broch, and D. B. Johnson, "Lessons from a full-scale multihop wireless ad hoc network testbed," IEEE Personal Communications, vol. 8, no. 1, pp. 8-15, February 2001.

[10] C. Perkins, E. Belding-Royer, and S. Das, "Ad hoc On-Demand Distance Vector (AODV) Routing," RFC 3561 (Experimental), July 2003.

[11] D. B. Johnson, D. A. Maltz, and J. Broch, DSR: The Dynamic Source Routing Protocol for Multi-Hop Wireless Ad Hoc Networks, ser. Ad Hoc Networking. Addison-Wesley, 2001, ch. 5, pp. 412-420.
[12] R. S. Gray, D. Kotz, C. Newport, N. Dubrovsky, A. Fiske, J. Liu, C. Masone, S. McGrath, and Y. Yuan, "Outdoor experimental comparison of four ad hoc routing algorithms," in Proc. MSWiM '04, 2004, pp. $220-229$.

[13] G. Anastasi, E. Borgia, M. Conti, and E. Gregori, "IEEE 802.11b ad hoc networks: Performance measurements," Cluster Computing, vol. 8, no. 2-3, pp. 135-145, 2005.

[14] V. Kawadia and P. R. Kumar, "Experimental investigations into tcp performance over wireless multihop networks," in Proc. of E-WIND-2005, 2005, pp. 2934.

[15] T. Clausen, G. Hansen, L. Christensen, and G. Behrmann, "The optimized link state routing protocol, evaluation through experiments and simulation," in Proc. of IEEE Symposium on Wireless Personal Mobile Communications, September 2001, http://hipercom.inria.fr/olsr/wpmc01.ps.

[16] D. Johnson and G. Hancke, "Comparison of two routing metrics in OLSR on a grid based mesh network," Ad Hoc Networks, vol. 7, pp. 374 - 387, 2009.

[17] G. De Marco, M. Ikeda, T. Yang, and L. Barolli, "Experimental performance evaluation of a pro-active adhoc routing protocol in out- and indoor scenarios," in Proc. AINA-2007, Canada, May 2007, pp. 7-14.

[18] T. Clausen and P. Jacquet, "Optimized link state routing protocol (OLSR)," RFC 3626 (Experimental), 2003.

[19] D. S. J. D. Couto, D. Aguayo, J. Bicket, and R. Morris, "A high-throughput path metric for multi-hop wireless routing," in Proc. MobiCom-2003, 2003, pp. 134-146.

[20] “Rt2x00 project," http://rt2x00.serialmonkey.com/.

[21] A. Botta, A. Dainotti, and A. Pescape, "Multi-protocol and multi-platform traffic generation and measurement," INFOCOM 2007 DEMO Session, May 2007.

[22] M. Ikeda, L. Barolli, G. De Marco, T. Yang, and A. Durresi, "Experimental and simulation evaluation of OLSR protocol for mobile ad-hoc networks," in Proc. NBiS-2008, Turin, Italy, September 2008, pp. 111-121. 